\title{
HerMES: THE REST-FRAME UV EMISSION AND A LENSING MODEL FOR THE $z=6.34$ LUMINOUS DUSTY STARBURST GALAXY HFLS3
}

\author{
Asantha Cooray ${ }^{1}$, Jae Calanog ${ }^{1}$, Julie L. Wardlow ${ }^{2}$, J. Bock ${ }^{3,4}$, C. Bridge ${ }^{3}$, D. Burgarella ${ }^{5}$, R. S. Bussmann ${ }^{6}$, \\ C. M. Casey ${ }^{1}$, D. Clements ${ }^{7}$, A. Conley ${ }^{8}$, D. Farrah ${ }^{9}$, H. Fu ${ }^{10}$, R. Gavazzi ${ }^{11}$, R. J. Ivison ${ }^{12,13}$, N. La Porte ${ }^{14}$, B. Lo Faro ${ }^{15}$, \\ Brian Ma ${ }^{1}$, G. Magdis ${ }^{16}$, S. J. Oliver ${ }^{17}$, W. A. Osage ${ }^{1}$, I. Pérez-Fournon $^{18}$, D. Riechers ${ }^{6}$, D. Rigopoulou ${ }^{16}$, \\ DOUGlas SCOTT ${ }^{19}$, M. VIERO ${ }^{1}$, AND D. WATSON ${ }^{2}$ \\ ${ }^{1}$ Center for Cosmology, Department of Physics and Astronomy, University of California, Irvine, CA 92697, USA \\ ${ }^{2}$ Dark Cosmology Centre, Niels Bohr Institute, University of Copenhagen, Juliane Maries Vej 30, DK-2100 Copenhagen, Denmark \\ ${ }^{3}$ California Institute of Technology, 1200 East California Boulevard, Pasadena, CA 91125, USA \\ ${ }^{4}$ Jet Propulsion Laboratory, 4800 Oak Grove Drive, Pasadena, CA 91109, USA \\ ${ }^{5}$ Laboratoire d'Astrophysique de Marseille, Aix-Marseille University, CNRS, F-13013 Marseille, France \\ ${ }^{6}$ Department of Astronomy, Space Science Building, Cornell University, Ithaca, NY 14853-6801, USA \\ ${ }^{7}$ Astrophysics Group, Imperial College London, Blackett Laboratory, Prince Consort Road, London SW7 2AZ, UK \\ ${ }^{8}$ Department of Astrophysical and Planetary Sciences, CASA 389-UCB, University of Colorado, Boulder, CO 80309, USA \\ ${ }^{9}$ Department of Physics, Virginia Tech, Blacksburg, VA 24061, USA \\ ${ }^{10}$ Department of Physics \& Astronomy, University of Iowa, Iowa City, IA 52242, USA \\ ${ }^{11}$ Institut d'Astrophysique de Paris, UMR 7095, CNRS, UPMC Univ. Paris 06, 98 bis boulevard Arago, F-75014 Paris, France \\ 12 European Southern Observatory, Karl Schwarzschild Strasse 2, D-85748 Garching, Germany \\ ${ }^{13}$ Institute for Astronomy, University of Edinburgh, Royal Observatory, Blackford Hill, Edinburgh EH9 3HJ, UK \\ ${ }^{14}$ Pontificia Universidad Caólica de Chile, Departamento de Astronomía y Astrofísica, Casilla 306, Santiago 22, Chile \\ ${ }^{15}$ Aix-Marseille Université, CNRS, LAM (Laboratoire d'Astrophysique de Marseille) UMR7326, F-13388 Marseille, France \\ ${ }^{16}$ Department of Astrophysics, Denys Wilkinson Building, University of Oxford, Keble Road, Oxford OX1 3RH, UK \\ ${ }^{17}$ Astronomy Centre, Department of Physics \& Astronomy, University of Sussex, Brighton BN1 9QH, UK \\ ${ }_{18}$ Instituto de Astrofísica de Canarias (IAC), E-38200 La Laguna, Tenerife, Spain \\ ${ }^{19}$ Department of Physics \& Astronomy, University of British Columbia, 6224 Agricultural Road, Vancouver, BC V6T 1Z1, Canada \\ Received 2014 April 6; accepted 2014 May 16; published 2014 July 1
}

\begin{abstract}
We discuss the rest-frame ultraviolet emission from the starbursting galaxy HFLS3 at a redshift of 6.34. The galaxy was discovered in Herschel/SPIRE data due to its red color in the submillimeter wavelengths from 250 to $500 \mu \mathrm{m}$. Keck/NIRC2 $\mathrm{K}_{s}$-band adaptive optics imaging data showed two potential near-IR counterparts near HFLS3. Previously, the northern galaxy was taken to be in the foreground at $z=2.1$, while the southern galaxy was assumed to be HFLS3's near-IR counterpart. The recently acquired Hubble/WFC3 and Advanced Camera for Surveys (ACS) imaging data show conclusively that both optically bright galaxies are in the foreground at $z<6$. A new lensing model based on the Hubble imaging data and the millimeter-wave continuum emission yields a magnification factor of $2.2 \pm 0.3$, with a $95 \%$ confidence upper limit on the magnification of 3.5 . When corrected for lensing, the instantaneous star formation rate is $1320 M_{\odot} \mathrm{yr}^{-1}$, with the $95 \%$ confidence lower limit around $830 M_{\odot} \mathrm{yr}^{-1}$. The dust and stellar masses of HFLS3 from the same spectral energy distribution (SED) models are at the level of $3 \times 10^{8} M_{\odot}$ and $\sim 5 \times 10^{10} M_{\odot}$, respectively, with large systematic uncertainties on assumptions related to the SED model. With Hubble/WFC3 images, we also find diffuse near-IR emission about $0.5 \operatorname{arcsec}(\sim 3 \mathrm{kpc})$ to the southwest of HFLS3 that remains undetected in the ACS imaging data. The emission has a photometric redshift consistent with either $z \sim 6$ or a dusty galaxy template at $z \sim 2$.
\end{abstract}

Key words: galaxies: high-redshift - galaxies: starburst - gravitational lensing: strong - infrared: galaxies submillimeter: galaxies

Online-only material: color figures

\section{INTRODUCTION}

The unexpected discovery of HFLS3 (HerMES J170647.8+ $584623)$ at a redshift of $6.3369 \pm 0.0009$ in Herschel Space Observatory (Pilbratt et al. 2010) has led to the possibility that massive starbursting galaxies could be an appreciable contributor to the star formation rate (SFR) density of the universe during the epoch of reionization (Riechers et al. 2013). The galaxy was first identified in the Herschel MultiTiered Extragalactic Survey (HerMES $;^{20}$ Oliver et al. 2012) as a high-redshift candidate due to its "red" color in the SPIRE (Griffin et al. 2010) data, with $S_{500} / S_{350} \sim 1.45$ and $S_{500} \sim 47 \pm 3 \mathrm{mJy}$. The redshift of HFLS3 was secured

\footnotetext{
${ }^{20}$ http://hermes.sussex.ac.uk
}

through the detection of more than 20 individual molecular and atomic lines at far-IR/submillimeter wavelengths with ground-based interferometers. HFLS3 was found to be luminous $\left(L_{\mathrm{IR}}=(3.4 \pm 0.3) \times 10^{13} L_{\odot}\right)$, gas-rich $\left(M_{\mathrm{gas}} \sim 10^{11} M_{\odot}\right)$, and dusty $\left(T_{\mathrm{d}}=49 \pm 2 \mathrm{~K}\right)$. The instantaneous SFR implied by the above total IR luminosity (Kennicutt 1998) is around $2900 M_{\odot} \mathrm{yr}^{-1}$ for a Chabrier (2003) IMF. It is also the highestredshift submillimeter galaxy (SMG) known to date, potentially probing the earliest formation epoch of dust in the universe (for a recent review of SMGs and dusty star-forming galaxies in general; see Casey et al. 2014).

One complication of interpreting the properties of HFLS3 is that it was found to be $\sim 0.5$ to the south of a $z=2.09$ galaxy (Figure 1), identified by Keck/NIRC2 $K$-band AO imaging and Keck/LRIS spectroscopy. This suggests some 

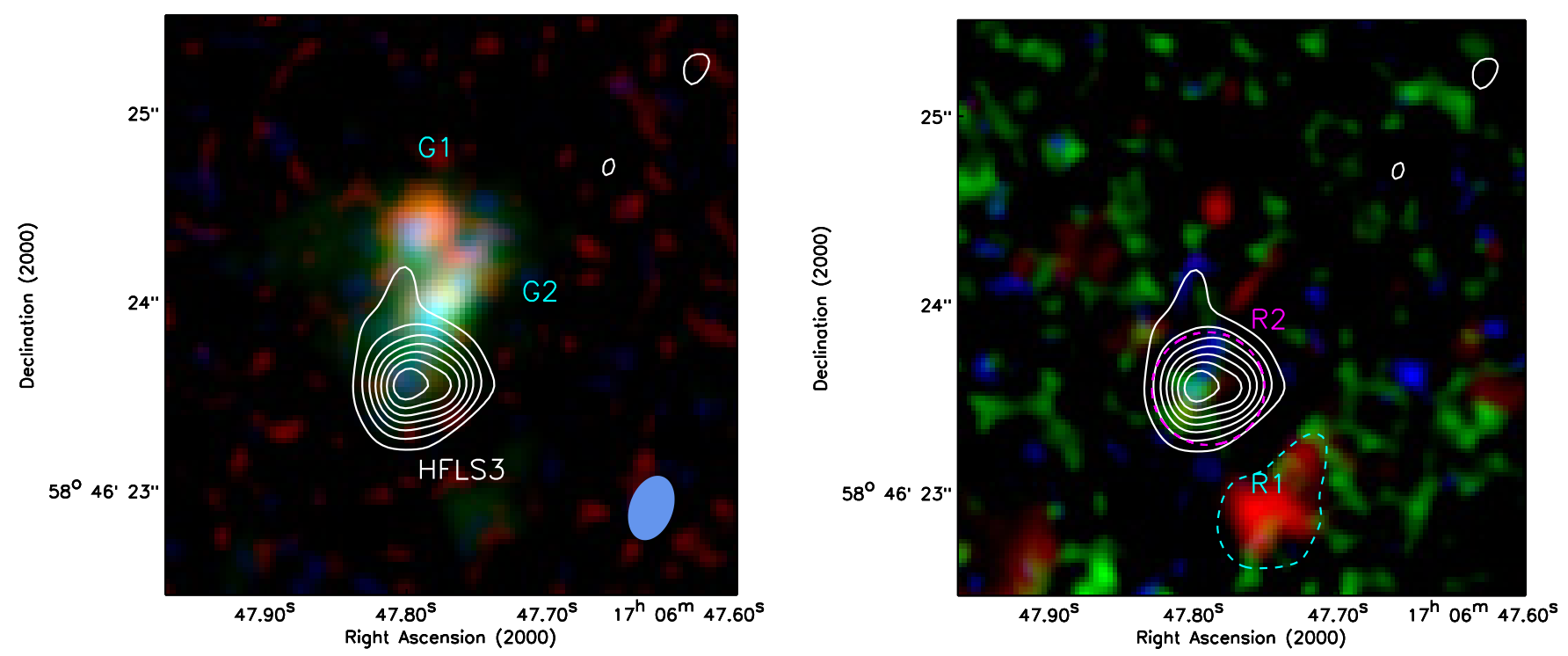

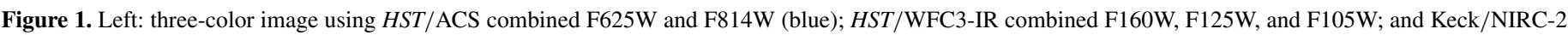

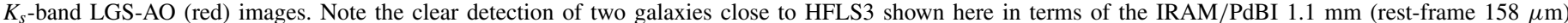

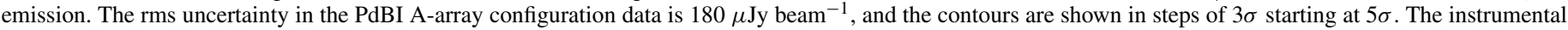

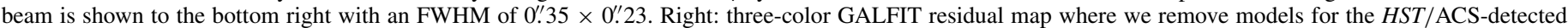

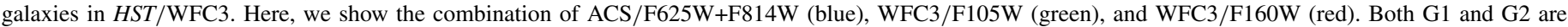

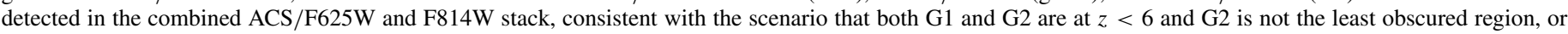

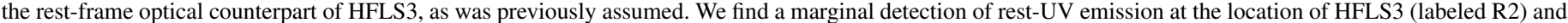

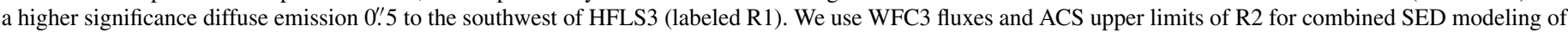

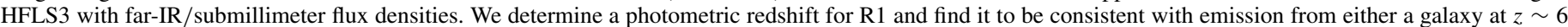
or a dusty galaxy at $z \sim 2$.

(A color version of this figure is available in the online journal.)

possibility that the flux density of HFLS3 is enhanced by gravitational lensing with a magnification factor $\mu_{\text {lens }}$. Due to the steepness of the SMG number counts and their high redshifts and the corresponding high magnification bias, submillimeter surveys are known to be highly sensitive to gravitational lensing modifications (Blain 1996; Perrotta et al. 2002; Negrello et al. 2007; Paciga et al. 2009). At the bright end of the number counts at wavelengths longer than $350 \mu \mathrm{m}$, lensed SMGs appear as a power law distinct from the intrinsic counts (e.g., Negrello et al. 2010; Wardlow et al. 2013; Vieira et al. 2013). At $z>4$, we expect the lensing fraction to be substantial for current generation surveys, where the flux density limit for the source detection is relatively high. An example of a high-efficiency lensing selection at $z>3$ is the bright SMG sample from the South Pole Telescope at $1.4 \mathrm{~mm}$ (Vieira et al. 2013; Weiß et al. 2013). If lensing is a statistically important correction to the flux densities of high-redshift SMGs, we expect them to be discovered near foreground galaxies and groups. Such a close association with a foreground galaxy is consistent with the existing indications that a reasonable fraction of the $z>7$ Lyman-break dropouts are also magnified by $\mu_{\text {lens }} \sim$ few due to their closeness to foreground bright galaxies (Wyithe et al. 2011).

In the case of HFLS3, a possibility for lensing was expected since the Keck/LRIS spectroscopy showed emission lines corresponding to a foreground galaxy at $z=2.1$ within 1 arcsecond of the peak $1.1 \mathrm{~mm}$ continuum emission. The highresolution Keck/NIRC2 LGS-AO imaging data in the $\mathrm{K}_{s}$ band showed two galaxies within $1^{\prime \prime} .5$ of HFLS3. In Riechers et al. (2013), the northern component was taken to be the $z=2.1$ foreground galaxy, while the southern component, close to the peak $1.1 \mathrm{~mm}$ emission, was taken to be the rest-frame optical counterpart, or the least obscured part, of HFLS3. Under such an assumption, deblended NIRC2 and Spitzer/IRAC photometry suggested a stellar mass of $\sim 3.7 \times 10^{10} M_{\odot}$. Thus, HFLS3 is already a stellar mass-rich galaxy at $z=6.34$, while continuing to form stars at a very high rate of $>2000 M_{\odot} \mathrm{yr}^{-1}$.

The lack of multiple images of HFLS3 in millimeter-wave interferometric imaging data was inferred to imply that the lensing magnification factor is negligible, with $\mu_{\text {lens }}=1.5 \pm 0.7$ associated with lensing by the foreground galaxy to the north of the assumed rest-frame optical counterpart. Due to such a small magnification, a lensing correction to the properties of HFLS3 was not included in Riechers et al. (2013). However, the lensing magnification determination is subject to assumptions related to the counterpart identification and the location of foreground galaxies relative to the millimeter-wave emission. Since the true mass and SFR of HFLS3 are directly related to its cosmic rarity, a potential lensing correction is even more important when addressing whether HFLS3 is a rare source among the SMG sample or if it is a source typical of $z>4$ SMGs (Daddi et al. 2009; Coppin et al. 2010; Capak et al. 2011; Walter et al. 2012; Combes et al. 2012).

Here, we report Hubble/WFC3 and Advanced Camera for Surveys (ACS) imaging observations of HFLS3 in five filters from optical to near-IR wavelengths. We use these data to study the physical properties of HFLS3 by improving the lensing model and by identifying rest-frame optical/UV emission for a new estimate of the stellar mass of HFLS3. This paper is organized as follows. In the next section, we summarize the observations and the analysis. We discuss the counterpart identification and GaLFIT (Peng et al. 2002) models in Section 3. Our lens models and the magnification factor of HFLS3 are presented in Section 4. In Section 5, we present the modeling of optical to IR spectral energy distribution (SED) of foreground galaxies and the UV to far-IR SED of HFLS3. We present 
a discussion of our key results and the implications for the presence of massive, dusty starbursts galaxies at high redshifts in Section 6 and conclude with a summary in Section 7. For lensing and SED models, we assume the best-fit concordance cosmology consistent with WMAP-9 year and Planck data (Hinshaw et al. 2013; Planck Collaboration et al. 2013).

\section{HUBBLE SPACE TELESCOPE OBSERVATIONS}

HFLS3 was observed with Hubble/ACS and WFC3 in Cycle 21 (GO 13045; PI: Cooray) in order to understand the nature and environment of the current highest-redshift dusty starburst known from submillimeter survey data. The observations were carried out in F160W/F125W/F105W filters with WFC3 and in F814W and F625W with ACS over a total of six orbits. The imaging data reach $5 \sigma$ point-source depths of $m_{\mathrm{AB}}=26.0,26.3,25.9,27.0$, and 26.1 in F160W, F125W, F105W, F814W, and F625W, respectively. While the WFC3 imaging was aimed at detecting the rest-frame UV emission from HFLS3, the ACS imaging was aimed at establishing the exact location, size, and morphology of the nearby $z \sim 2.1$ galaxy for an improved lens model. The five-band photometry was aimed at completing the rest-UV SED of HFLS3 to improve the stellar mass estimate once combined with Keck/NIRC2 $\mathrm{K}_{s}$ and Spitzer/IRAC photometry. Here, we focus on properties of HFLS3, but another study will discuss the environment of HFLS3 (N. Laporte et al., in preparation). The HST data are also useful for a near-IR counterpart search of SCUBA-2 sources detected in the HFLS3 field (Robson et al. 2014).

The Hubble data were analyzed with the standard tools. For WFC3 imaging data, we make use of CALWFC3 in the IRAF.STSDAS pipeline for flat-fielding and cosmic-ray rejection. Individual exposures in each of the filters were combined with AsTRODRIZZLE (Fruchter \& et al. 2010), and we produced images at a pixel scale of 0 .'06 from the native scale of 0.13 per pixel. For flux calibration, we made use of the latest zeropoints from STScI, with values of 26.27, 26.26, and 25.96 in F105W, F125W, and F160W, respectively. Similarly, Hubble/ ACS imaging data were flat-fielded, cosmic-ray-rejected, and charge transfer efficiency (CTE)-corrected with the pipeline CALACS (version 2012.2). Exposures were remapped with AsTRODRIZZLE to a pixel scale of 0'03. The ACS zero-points used from an online tool are 25.94 and 25.89 for F814W and F625W, respectively.

The final Hubble mosaics were astrometrically calibrated to the wider Sloan Digital Sky Survey (SDSS) frame with an overall rms uncertainty, relative to SDSS, of less than 0.'05. This astrometric calibration involved more than 60 galaxies and stars. The previous Keck/NIRC2 imaging data, due to the limited field of view of $40^{\prime \prime}$ in the highest-resolution NIRC2 imaging data used for LGS/AO observations, had large astrometric errors as astrometry was determined based on two bright sources that were also detected in 2MASS. Once the HST frames are calibrated, we fixed the astrometry of Keck/NIRC2 image with close to 10 fainter sources detected in both WFC3 and NIRC2 images. This astrometric recalibration resulted in a small (0!'1) shift to the optical sources relative to the peak PdBI/1.1 mm emission from HFLS3, as can be seen by comparing Figure 1 here with Figure 3 of Riechers et al. (2013). There is still an overall systematic uncertainty in the relative astrometry between IRAM/PdBI image and Hubble/Keck images of about 0 !' 1 , with this value possibly as high as 0.3 in an extremely unlikely scenario. We account for such a systematic offset in the lens model by allowing the peak $1.1 \mathrm{~mm}$ flux to have an offset from the two lens galaxies with a value as high as 0.3.

As shown in Figure 1 (left panel), we detect optical emission from more than one galaxy near HFLS3 (galaxies labeled G1 and $\mathrm{G} 2$ ). This is similar to what was previously reported with Keck/ NIRC2 LGS-AO imaging data, with the southern component (G2) taken to be the rest-frame optical counterpart to HFLS3 (Riechers et al. 2013). If this assumption is correct, we expect the southern component to be invisible in the shortest-wavelength images, as it is a Lyman dropout at wavelengths shorter than $8900 \AA$. Here, however, we have detected both galaxies in Hubble/ACS images, establishing that G2 is a galaxy at $z<5$. Since these Hubble observations, we have reanalyzed the Keck/ LRIS spectrum shown in Riechers et al. (2013) with $z=2.1$ CIV (1549 ^) and O III] $(1661,1666 \AA)$ emission lines within $1^{\prime \prime}$ of HFLS3. We now find some marginal evidence that this emission is extended, consistent with the scenario that more than one galaxy may be contributing to the emission lines. A further confirmation of the redshift of $\mathrm{G} 2$ will require additional spectroscopic observations or UV imaging data where $z \sim 2$ galaxies would be Lyman dropouts. For simplicity, hereafter, we assume that both $\mathrm{G} 1$ and $\mathrm{G} 2$ are at the same redshift of 2.1. The SED modeling we discuss later is consistent with this assumption.

\section{REST-FRAME UV FLUXES OF HFLS3}

We use the publicly available software GALFIT (Peng et al. 2002) to model the surface brightness profiles of Hubbledetected galaxies near HFLS3 and to see if there is any excess emission in WFC3 data relative to the ACS images. Using GALFIT on the individual Hubble/ACS and WFC3 frames proved to be difficult because the output models tend to overfit regions of low signal in which HFLS3 is expected to reside. To remedy this, we stacked the HST/ACS in two bands to increase the signal-tonoise ratio and to model the foreground galaxies in the combined F625W and F814W images. Under the assumption that the stacked model best represents the two foreground galaxies, we then subtracted the stacked model from individual HST/ACS and WFC3 frames, with the flux density at each wavelength allowed to vary as an overall normalization in GALFIT models. Any excess in WFC3 relative to ACS would suggest the presence of detectable rest-UV emission from HFLS3. As shown in Figure 1 (right panel), we find excess emission primarily 0.5 to the southwest of HFLS3 (labeled R1). We also find some marginal evidence for excess emission near the $1.1 \mathrm{~mm}$ peak (labeled R2), with detection levels between 2.5 to $3.2 \sigma$. In Table 1, we summarize GALFIT and other intrinsic properties of the two foreground galaxies G1 and G2, as well as the residual emission R1 and R2, with R2 emission assumed to be from HFLS3. We also use the latter for a combined UV to far-IR SED modeling with MAGPHYs (da Cunha et al. 2008). We also model the SED of R2 to determine its photometric redshift and address its association with HFLS3.

\section{LENS MODELING}

We use the publicly available software GRAVLENS (Keeton 2001) to generate the lens model. As the background source is not multiply imaged, and remains undetected in the rest-frame optical, we measure the goodness of fit of the model using the highest-resolution IRAM/PdBI $1.1 \mathrm{~mm}$ continuum emission map from Riechers et al. (2013). This map is currently our highest-resolution view of HFLS3, and the source is resolved 
Table 1

IR Properties of HFLS3 and Nearby Galaxies

\begin{tabular}{|c|c|c|}
\hline Quantity & Value & Ref \\
\hline \multicolumn{3}{|c|}{ G1 } \\
\hline R.A. & $17: 06: 47.80$ & ACS $I_{814}$-band \\
\hline Decl. & $+58: 46: 24.33$ & ACS $I_{814}$-band \\
\hline Redshift & 2.019 & Riechers et al. (2013) \\
\hline ACS/F625W & $27.01 \pm 0.14(\mathrm{AB} \mathrm{mag})$ & Photometry \\
\hline ACS/F814W & $26.17 \pm 0.12(\mathrm{AB} \mathrm{mag})$ & Photometry \\
\hline WFC3/F105W & $25.27 \pm 0.12(\mathrm{AB} \mathrm{mag})$ & Photometry \\
\hline WFC3/F125W & $25.27 \pm 0.04(\mathrm{AB} \mathrm{mag})$ & Photometry \\
\hline WFC3/F160W & $24.57 \pm 0.09(\mathrm{AB} \mathrm{mag})$ & Photometry \\
\hline $\mathrm{NIRC} 2 / \mathrm{K}_{\mathrm{s}}$-band & $23.94 \pm 0.04(\mathrm{AB} \mathrm{mag})$ & Photometry \\
\hline$R_{\mathrm{E}}$ & $0^{\prime \prime} 05_{-00^{+\prime \prime}, 01}^{\prime \prime}$ & lens model \\
\hline$M_{\mathrm{E}}$ & $\left(1.2_{-0.2}^{+6.4}\right) \times 10^{9} M_{\odot}$ & lens model \\
\hline$R_{\mathrm{e}}$ & $0{ }^{\prime \prime} 9 \pm 0.9$ & GALFIT \\
\hline & $7.1 \pm 2.3 \mathrm{kpc}$ & \\
\hline$\epsilon$ & $0.48 \pm 0.02$ & GALFIT \\
\hline $\mathrm{PA}_{\mathrm{d}}$ & $(88 \pm 2)^{\circ}$ & GALFIT \\
\hline$n$ (Sérsic) & $4.3 \pm 0.8$ & GALFIT \\
\hline$M_{\star}$ & $\sim 8 \times 10^{8} M_{\odot}$ & SED (HYPERZ) \\
\hline$A_{V}$ & $\sim 3.4 \mathrm{mag}$ & SED (HYPERZ) \\
\hline$L_{V}($ extinction corrected $)$ & $\sim 3 \times 10^{11} L_{\odot}$ & SED (HYPERZ) \\
\hline \multicolumn{3}{|c|}{$\mathrm{G} 2$} \\
\hline R.A. & $17: 06: 47.77$ & ACS $I_{814}$-band \\
\hline Decl. & $+58: 46: 23.95$ & ACS $I_{814}$-band \\
\hline ACS/F625W & $25.42 \pm 0.13(\mathrm{AB} \mathrm{mag})$ & Photometry \\
\hline ACS/F814W & $25.50 \pm 0.16(\mathrm{AB} \mathrm{mag})$ & Photometry \\
\hline WFC3/F105W & $25.22 \pm 0.13(\mathrm{AB} \mathrm{mag})$ & Photometry \\
\hline WFC3/F125W & $24.68 \pm 0.05(\mathrm{AB} \mathrm{mag})$ & Photometry \\
\hline WFC3/F160W & $24.59 \pm 0.13(\mathrm{AB} \mathrm{mag})$ & Photometry \\
\hline $\mathrm{NIRC} 2 / \mathrm{K}_{\mathrm{s}}$-band & $23.72 \pm 0.09(\mathrm{AB} \mathrm{mag})$ & Photometry \\
\hline$R_{\mathrm{E}}$ & $0^{\prime \prime} 15^{+0^{\prime \prime} \cdot 02}$ & lens model \\
\hline$M_{\mathrm{E}}$ & $\left(1.2_{-0.1}^{+0.2}\right) \times 10^{10} M_{\odot}$ & lens model \\
\hline$R_{\mathrm{e}}$ & $0^{\prime \prime} 34 \pm 0.0^{\prime \prime} 01$ & GALFIT \\
\hline & $2.8 \pm 0.1 \mathrm{kpc}$ & \\
\hline$\epsilon$ & $0.63 \pm 0.01$ & GALFIT \\
\hline $\mathrm{PA}_{\mathrm{d}}$ & $(-30 \pm 1)^{\circ}$ & GALFIT \\
\hline$n$ (Sérsic) & $0.98 \pm 0.03$ & GALFIT \\
\hline$M_{\star}$ & $1 \times 10^{10} M_{\odot}$ & SED (HYPERZ) \\
\hline$A_{V}$ & $1.20 \mathrm{mag}$ & SED (HYPERZ) \\
\hline$L_{V}($ extinction corrected $)$ & $4 \times 10^{10} L_{\odot}$ & SED (HYPERZ) \\
\hline \multicolumn{3}{|c|}{ HFLS3 } \\
\hline R.A. & $17: 06: 47.80$ & Riechers et al. (2013) \\
\hline Decl. & $+58: 46: 23.51$ & Riechers et al. (2013) \\
\hline Redshift & $6.3369 \pm 0.0009$ & Riechers et al. (2013) \\
\hline ACS/F625W & $>27.01(\mathrm{AB}$ mag $)$ & Photometry \\
\hline ACS/F814W & $>28.20(\mathrm{AB} \mathrm{mag})$ & Photometry \\
\hline WFC3/F105W & $>27.58(\mathrm{AB} \mathrm{mag})$ & Photometry \\
\hline WFC3/F125W & $27.02 \pm 0.35(\mathrm{AB} \mathrm{mag})$ & Photometry \\
\hline WFC3/F160W & $27.06 \pm 0.38$ & Photometry \\
\hline $\mathrm{NIRC} 2 / \mathrm{K}_{\mathrm{s}}$-band & $25.64 \pm 0.50$ & Photometry \\
\hline \multirow{3}{*}{$\begin{array}{l}\mu_{\text {lens }} \\
\Theta_{s 1}\end{array}$} & $2.2 \pm 0.3$ & Two-component model \\
\hline & $0^{\prime \prime} .5 \pm 0 . .1$ & Component 1 \\
\hline & $2.6 \pm 0.7 \mathrm{kpc}$ & \\
\hline \multirow[t]{2}{*}{$\Theta_{s 2}$} & $0.3_{-0.0_{1}^{\prime \prime}}^{+0^{\prime \prime} 2}$ & Component 2 \\
\hline & $1.6_{-0.6}^{+1.2} \mathrm{kpc}$ & \\
\hline$F_{2} / F_{1}$ & $0.3_{-0.2}^{+0.4}$ & Flux ratio \\
\hline$M_{\text {dust }}$ & $3 \times 10^{8} M_{\odot}$ & SED (MAGPHYS) \\
\hline $\mathrm{SFR}_{\text {int }}$ & $1320 M_{\odot} \mathrm{yr}^{-1}$ & Kennicutt (1998) \\
\hline$\langle\mathrm{SFR}\rangle_{100 \mathrm{Myr}}$ & $654_{-90}^{+104} M_{\odot} \mathrm{yr}^{-1}$ & SED (MAGPHYS) \\
\hline$M_{\star}$ & $\sim 5 \times 10^{10} M_{\odot}$ & SED (MAGPHYS) \\
\hline
\end{tabular}


Table 1

(Continued)

\begin{tabular}{lcc}
\hline \hline Quantity & Value & Ref \\
\hline$A_{V}$ & $3.6 \mathrm{mag}$ & SED (MAGPHYS) \\
$L_{V}$ (extinction corrected) & $\sim 4 \times 10^{12} L_{\odot}$ & SED (MAGPHYs) \\
\hline & $\mathrm{R} 1$ & \\
\hline R.A. & $17: 06: 47.76$ & WFC3 $H_{160}$-band \\
Decl. & $+58: 46: 22.87$ & WFC3 $H_{160}$-band \\
ACS/F625W & $>27.01(\mathrm{AB} \mathrm{mag})$ & Photometry \\
ACS/F814W & $>26.85(\mathrm{AB} \mathrm{mag})$ & Photometry \\
WFC3/F105W & $26.68 \pm 0.28(\mathrm{AB} \mathrm{mag})$ & Photometry \\
WFC3/F125W & $26.20 \pm 0.14(\mathrm{AB} \mathrm{mag})$ & Photometry \\
WFC3/F160W & $26.03 \pm 0.15(\mathrm{AB} \mathrm{mag})$ & Photometry \\
NIRC2/K - band & $26.30 \pm 0.92(\mathrm{AB} \mathrm{mag})$ & Photometry \\
\hline
\end{tabular}

Notes. We assume $z_{\mathrm{G} 1}=z_{\mathrm{G} 2}$. For non-detections, flux density upper limits are given at $3 \sigma$. For HFLS3, flux densities are not corrected for lensing magnification, but physical properties are assuming $\mu=2.2$. We do not list IRAC flux densities due to large uncertainties in deblending and the separation of total flux density to the four source components.

in these data. The magnification factor we determine here with lens modeling is the value for the millimeter-wave continuum emission. It could be that HFLS3 will be subject to differential magnification, with different emission components within the galaxy subject to different magnification factors (e.g., Serjeant 2012; Hezaveh et al. 2012). This is especially true if the different components associated with HFLS, such as dust, gas, and stellar mass, have peak intensities that are offset from each other, as in the case of a complex merging galaxy system.

To simplify the lens modeling, we use singular isothermal ellipsoidal (SIE) models to fit for the Einstein radius and positions of the two lens galaxies. The position angles and ellipticities for G1 and G2 are fixed to the values derived from profile fitting using GALFIT, but their masses are allowed to vary freely. The relative positions of $\mathrm{G} 1$ and $\mathrm{G} 2$ are also kept fixed to Hubble/ACS-stack measurements, though we do allow the optical galaxy positions to vary relative to the peak location of the $1.1 \mathrm{~mm}$ continuum emission. For the source plane description of HFLS3, we considered two options: a single source for HFLS3 and a two-component model for HFLS3. The latter is motivated by the fact that the highest-resolution [C II] line emission may involve two velocity components separated by about $400 \mathrm{~km} \mathrm{~s}^{-1}$ (Riechers et al. 2013). In both these cases, the background source(s) is/are modeled with free parameters for the positions and effective radii. For simplicity, we assume Gaussian circular profiles with a fixed Sersic index of 0.5 . The effective radii in the source plane are allowed to vary in the range of 0 '.005 to $2^{\prime \prime}$. 0 , with the upper end at a value higher than the measured size of the $1.1 \mathrm{~mm}$ continuum emission in the PdBI image. In the case of the two-component model, the flux ratio between the two background components is also left as a free parameter. Hence, the lens model fits for a total of five free parameters for the case with one component for HFLS3 or nine free parameters for the case with two components. We take this two-component model for the background source as a default model here, though our conclusions do not change if we adopt the single-component model.

In the lens modeling procedure, we output a lensed image as would be observed at $1.1 \mathrm{~mm}$. However, to compare with the data, we convolve that image with the PdBI beam before calculating the $\chi^{2}$ value. This process is iterated over a wide parameter space using the IDL routine AMOEBA_SA, which uses a downhill simplex algorithm and simulated annealing to perform multidimensional minimization. We use a circle of a radius $1^{\prime \prime} .5$ centered on the peak pixel value of the PdBI $1.1 \mathrm{~mm}$ image to measure parameter errors from uncorrelated noise. For each iteration, the $1.1 \mathrm{~mm}$ magnification factor we quote, $\mu_{\text {lens }}$, is calculated by simply summing up all the pixel values in the image plane and dividing it by the sum of the pixel values in the source plane.

Figure 2 shows the best-fit model for the two scenarios with one and two components. In the case of two components, we determine $\mu_{\text {lens }}=2.2 \pm 0.3$. The two components have effective radii of $0.5 \pm 0.1 \mathrm{kpc}$ and $0.3 \pm 0.1$. The best-fit model has $\chi^{2}$ and number of degrees of freedom (Ndof) values of 9929 and 7835, respectively. For reference, the model with a single component for the background source has $\mu_{\text {lens }}=2.0_{-0.1}^{+0.9}$, an effective radius of $0.6 \pm 0.1 \mathrm{kpc}$, and $\chi^{2} /$ Ndof of $100552 /$ 7839. The lensing masses are $M_{\text {lens }}=1.2_{-0.2}^{+6.4} \times 10^{9} M_{\odot}$ and $1.2_{-0.1}^{+0.2} \times 10^{10} M_{\odot}$ for G1 and G2, respectively, for the twocomponent model. We find masses consistent within these errors for the case when HFLS3 is described by a single component. The lensing model is mostly sensitive to the mass of G2, while G1, the galaxy farthest from HFLS3, remains as a minor contribution to the lens model. Therefore, the mass of G1 is less constrained in the lens model. The best-fit Einstein radius for $\mathrm{G} 1$ that we find with the value of $0 .^{\prime} 05^{+0^{\prime \prime}, 06}$ is barely above the lower value of $0{ }^{\prime} .01$ for the Einstein radius that we placed on the parameter ranges. We emphasize that the lens model presented here does not require the presence of two lenses in the foreground or two sources in the background to fit the data.

Note that we have assumed the redshifts of G1 and G2 are the same in the lens model. The lens magnifications discussed here are insensitive to the exact assumption related to the redshifts of G1 and G2 as a change in redshift is degenerate with their lensing masses. Our lens model also assumes a single lensing plane and do not account for multiple-plane lensing if G1 and G2 are at two different redshifts. If the two galaxies are indeed at the same redshift, they could be part of a galaxy group. The mass we have determined then could have a contribution from the group potential and will be higher than the value implied by the stellar mass of these galaxies. Our lens models do show some evidence for such a possibility, but due to overall uncertainties in the stellar mass from SED fits, we cannot reliably confirm this with the current data. 

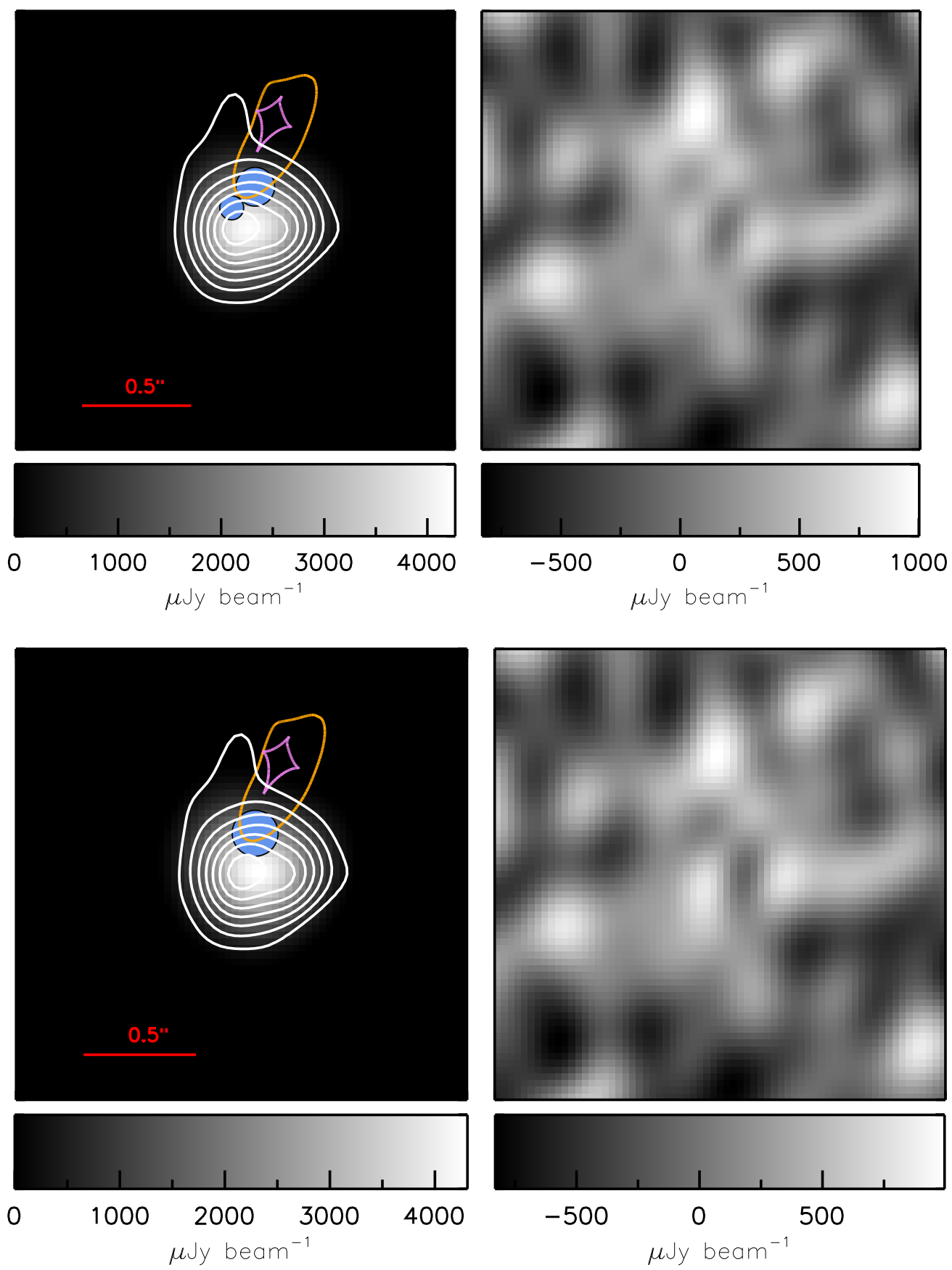

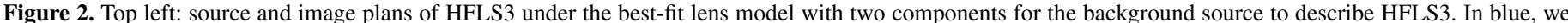

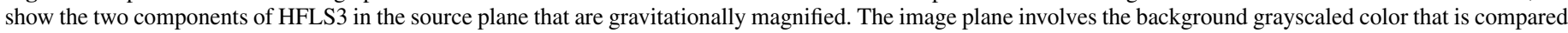

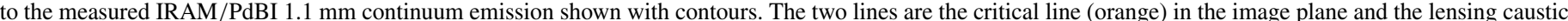

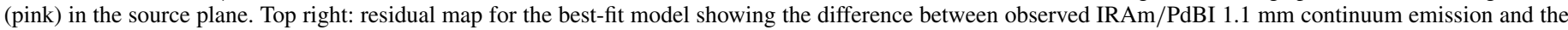

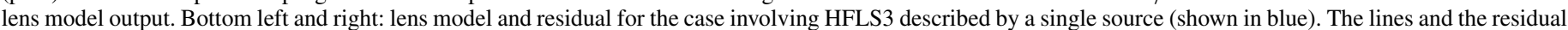
intensity to the right are the same as top two panels.

(A color version of this figure is available in the online journal.)

In addition to the best-fit lens model, we are also able to place a reliable upper limit on the lensing magnification of HFLS3 at $1.1 \mathrm{~mm}$. This is simply based on the fact that we have not detected a counter image to HFLS3, while large magnification factors usually result in image multiplication leading to a detectable counter image. Using the same modeling procedure as described above, and allowing for the model to vary over all ranges and including a relative astrometry as high as 0 '. 3 between $1.1 \mathrm{~mm}$ image and lens locations, we constructed the probability distribution function (PDF) of magnification for the two cases involving one and two source components to describe HFLS3 in the source plane. In Figure 3, we show the histogram where we highlight the 95\% confidence level upper limit on magnification such that $\mu_{\text {lens }}<3.5$ and 2.7 for the two cases with one and two components, respectively. The probability distribution functions also show that there is a strict lower limit to magnification. The case with $\mu_{\text {lens }}=1$, where HFLS3 is unlensed, is ruled out at more than $6 \sigma$. This is simply because of the fact that even a very small mass for G2, the galaxy closest to HFLS3, will result in some lensing magnification of HFLS3. 


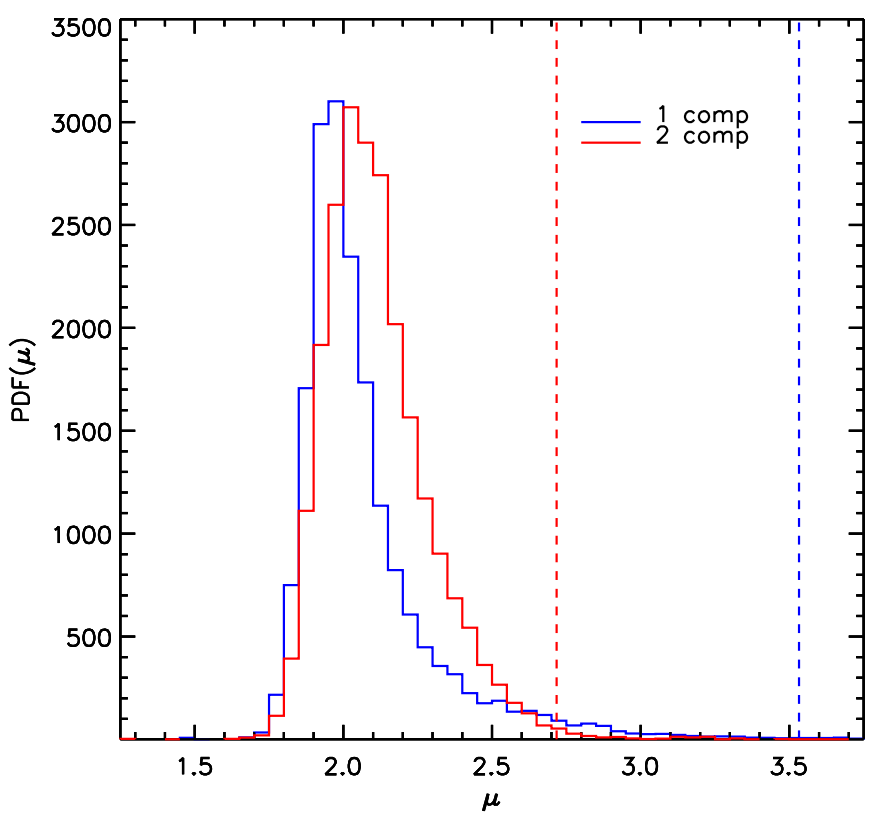

Figure 3. Probability distribution function of the lensing magnification $\mu_{\text {lens }}$ at $1.1 \mathrm{~mm}$ for HFLS3. We show two scenarios here for the case where HFLS3 is described by either a single (blue) or double (red) source in the source plane. The vertical lines show the $95 \%$ confidence level upper limit on the magnification. Note that in both scenarios there is also a strict lower limit for magnification with $\mu_{\text {lens }}>1.6$ at the $95 \%$ confidence level. The case with $\mu_{\text {lens }}=1$ is rejected at $>6 \sigma$ in both cases.

(A color version of this figure is available in the online journal.)

\section{SED MODELING}

We carry out SED modeling of both foreground and background galaxies using a combination of HYPERZ ${ }^{21}$ (Bolzonella et al. 2000) and MAgPhys (da Cunha et al. 2008). For SED modeling involving G1 and G2, we fix the redshift to the value determined from optical spectroscopy (Figure 5). We make use of Bruzual \& Charlot (2003) SED templates with a combination of single burst, constant, and exponentially declining star formation history ( $\tau$-models), with $\tau$ fixed at 1 Gyr ("E") and 5 Gyr ("Sb"). Internal reddening is included using the Calzetti et al. (2000) extinction law and allowing $A_{V}=0-5 \mathrm{mag}$ in steps of 0.2 . We also make use of the default Ly $\alpha$ forest following the prescription from Madau (1995). Given the parameters from the SED modeling (SED type, $\mathrm{A}_{V}$, age, etc.), we then make use of the Bruzual \& Charlot (2003) models to calculate the $H$-band mass-to-light ratio assuming a Chabrier initial mass function (IMF; Chabrier 2003). In the case of SED fits, we note that quoted error bars are the formal uncertainties and do not include systematic effects. In most of our modeling cases, it could very well be that uncertain systematics, such as on the choice of SED templates, dominate the error budget.

For G1 and G2, we find stellar masses of $8 \times 10^{8}$ and $1 \times 10^{10} M_{\odot}$, respectively. The ratio of stellar-to-lensing mass for two lensing galaxies at $z=2.1$ ranges from 0.66 to 0.85 . We find that significant dust attenuation is present in the northern galaxy G1, with $A_{V} \sim 3.4 \mathrm{mag}$. We do not find millimeter-wave emission from that galaxy in our deep $1.1 \mathrm{~mm}$ interferometric continuum emission data, ruling out a submillimeter bright dusty galaxy at this location. It could be that $\mathrm{G} 1$ is blended with another galaxy or that our assumption of 2.1 for the redshift is invalid. As our conclusions related to the

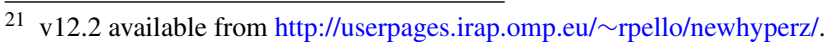

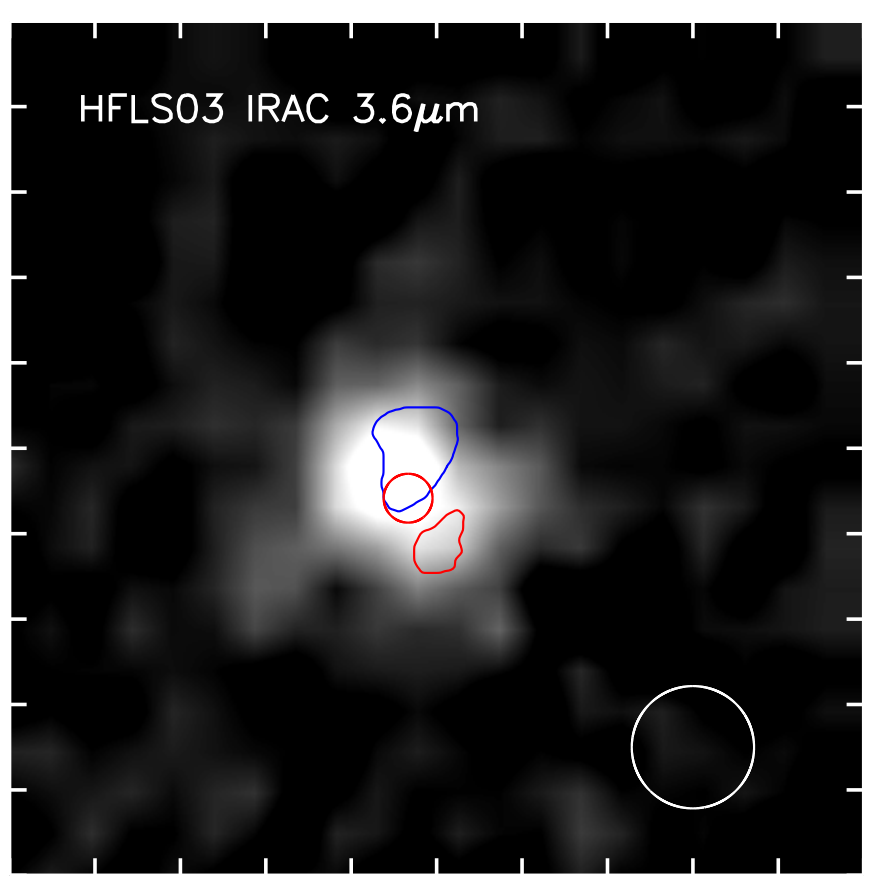

Figure 4. $10^{\prime \prime} \times 10^{\prime \prime}$ Spitzer/IRAC $3.6 \mu \mathrm{m}$ image from Riechers et al. (2013) showing a detected source. Each tick mark represents $2^{\prime \prime}$. The contours on the intensity scale show the regions of G1 and G2 (blue) and R1 and R2 (red). The IRAC PSF (FWHM = 1".5) is marked with a white circle on the bottom right. Note that the total flux density measured in IRAC is blended to multiple sources. We are able to measure the total flux density from G1 and G2. The residual after removing G1+G2 flux density is assumed to be from the new source R1 when attempting to determine its photometric redshift (Figure 5).

(A color version of this figure is available in the online journal.)

physical properties of the two foreground galaxies depend on their redshifts, we caution that the properties of these galaxies not be overinterpreted. Further deep imaging and spectroscopy should resolve some of the remaining puzzles in the data.

Given the nature and rarity of sources such as HFLS3 at $z>5$, it is useful to address the extent to which the recent Hubble/ACS and WFC3 imaging changes the underlying properties of this dusty, starburst galaxy. In order to establish the rest-frame UV fluxes of HFLS3, we made use of the ACS-based models of the two foreground galaxies to search for excess emission in the longer-wavelength data. Note that in ACS, $z>6$ emission should not appear since at those redshifts, galaxies will be dropping out of the band due to the Lyman limit. Using the ACS models on WFC3 data, we found marginal evidence, at around $3.5 \sigma$, for rest-frame UV emission at the PdBI $1.1 \mathrm{~mm}$ emission position in F125W (region marked as R2 in Figure 1, right panel). At the same location, we also found $2.8 \sigma$ residual emission in both the F160W and Keck/NIRC2 images. We consider these flux densities to be the rest-UV emission from HFLS3 itself.

Unfortunately, due to blending in the $\sim 2^{\prime \prime}$ PSF, we are not able to deconvolve the existing Spitzer/IRAC data to precisely determine the rest-frame optical flux densities of G1, G2, and HFLS3 separately (Figure 4). The IRAC flux densities for HFLS3 reported in Riechers et al. (2013) made use of a GALFIT model for G2 with Keck/NIRC2 image to deblend its contribution from the total. The residual flux densities are then those corresponding to G1 and HFLS3 in IRAC data and the total residual was assumed to be those of HFLS3, under the assumption that G1 is the near-IR counterpart of HFLS3. However, as discussed above, such an assumption no longer 


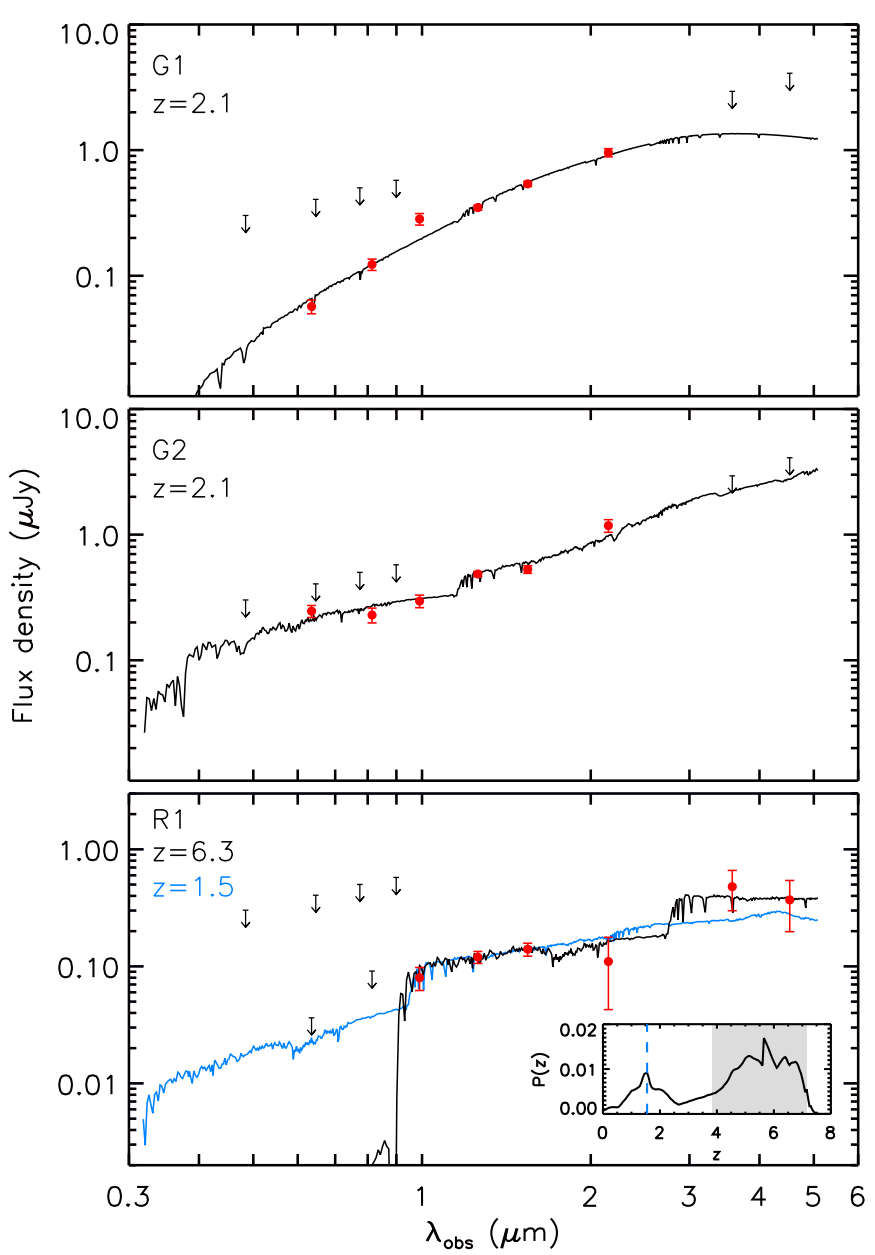

Figure 5. SEDs and best-fit HYPERZ models for optical to IR SEDs of G1 (top), G2 (middle), and R1 (bottom). For G1 and G2, we assume an optical redshift of 2.1, though we have yet to establish if the measured optical redshift applies to either G1 or G2, or to both. For R1, we allow the redshift to vary as part of the SED models, and the probability distribution function for the photometric redshift is shown in the inset to the bottom right of the panel. We find two solutions with one at high redshift consistent with $z \sim 6$ and a second, involving dusty galaxy templates, at $z \sim 1.3-2.3$. The $\chi^{2}$ values for the best-fit SEDs are 12.8 and 12.2 for G1 with the number of degrees of freedom (Ndof) at 11. For R1, the two SEDs show have $\chi^{2}$ values of $5.4(z \sim 6)$ and $6.1(z \sim 1.5)$ with an Ndof of 9 .

(A color version of this figure is available in the online journal.)

applies. Through the GALFIT model from Hubble/ACS data we are able to extract the total G1+G2 IRAC flux densities, but we are not able to separate that total to each of the two galaxies. Thus, in Figure 5, we show IRAC flux densities as upper limits for $\mathrm{G} 1$ and $\mathrm{G} 2$. We detect a residual after removing $\mathrm{G} 1+\mathrm{G} 2$, and in Figure 5, we assume that residual corresponds to R1. A fraction of that residual could also be from HFLS3 at the region marked as R2 in Figure 1 (right panel). We find that even such an upper limit is subject to assumptions related to GALFIT modeling in IRAC images, where multiple components exist within a single IRAC PSF. Thus, in Figure 6, we simply use the total flux density measured with IRAC as a conservative upper limit on the flux density of HFLS3 at 3.6 and $4.5 \mu \mathrm{m}$.

With the rest-UV fluxes for HFLS3 determined with Hubble/ WFC3 and Keck/NIRC2 data fluxes, we cover four orders of magnitude in wavelength from rest-frame UV to far-infrared (Figure 4). This SED of HFLS3 is fitted using MAGPHYS, where models are calibrated to reproduce ultraviolet-to-infrared SEDs of local, purely star-forming ultraluminous infrared galaxies

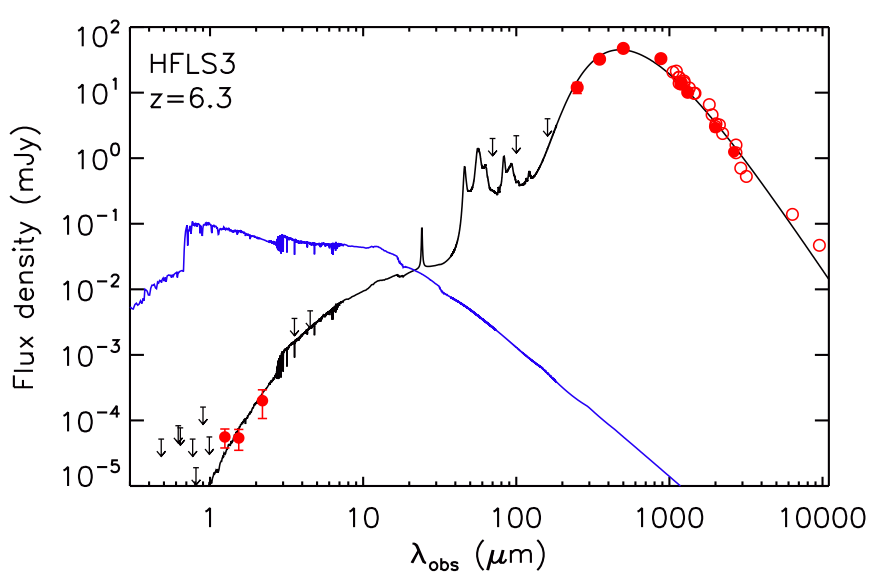

Figure 6. MAGPHYs (da Cunha et al. 2008) best-fit SED model of HFLS3 from rest-UV to far-infrared over four decades in wavelength (with a reduced $\chi^{2}$ value of 1.6). The blue line shows the unobscured template. For UV to submillimeter SED modeling, we make use of the far-IR/submillimeter data shown by solid symbols. Other measurements shown with open symbols involve non-standard bands that are not part of the filter and bandpass table of MAGPHYS. They were obtained as part of continuum measurements during atomic and molecular line measurements of HFLS3 with ground-based interferometers.

(A color version of this figure is available in the online journal.)

(ULIRGS; $10^{12}<L_{\mathrm{IR}} / L_{\odot}<10^{13}$ ). Such models, however, are based on the assumption that dust and stars are in a fully mixed medium. Massive, dusty starbursts at $z>2$ may not follow such mixing with differential obscuration causing biases in the combined UV to radio SED. For example, regions that are bright in the rest-frame optical may only be a small fraction of the regions that are bright in the far-infrared and submillimeter wavelengths. The use of MAGPHYs to model such complex galaxies may result in biased estimates of the physical parameters, but in the absence of other methods to study the combined SED, we have decided to use MAGPHYs here with appropriate caution.

The SED model assumes a Chabrier (2003) IMF that has a cutoff below $0.1 M_{\odot}$ and above $100 M_{\odot}$; using a Salpeter IMF instead gives stellar masses that are a factor of $\sim 1.7$ to 1.8 larger. With MAgPHYs-based SED models, we find that HFLS3, with rest-UV fluxes in the region marked as $\mathrm{R} 2$, shows significant dust attenuation with $A_{V} \sim 3.6 \mathrm{mag}$. Such attenuation is consistent with $z \sim 2$ ULIRGs and SMGs (e.g., Smail et al. 2004; Chapman et al. 2005; Geach et al. 2007; Swinbank et al. 2010; Wardlow et al. 2011; Hainline et al. 2011; Lo Faro et al. 2013). The best-fit MAGPHYs SED model is shown in Figure 6. The fit is dominated by the far-IR/submillimeter data, and the overall fit has a reduced $\chi^{2}$ value of 1.6.

\section{DISCUSSION}

The MagPhys SED models of HFLS3 described above lead to SFR, dust mass, and stellar mass, among other properties. As outlined in Riechers et al. (2013), the instantaneous SFR, using the FIR luminosity and assuming a Chabrier (2003) IMF to scale the Kennicutt (1998) relation, is $\sim 2900 M_{\odot} \mathrm{yr}^{-1}$. Using the MAGPHYs SED model, we find that the apparent SFR, averaged over the last $100 \mathrm{Myr}$, to be $1450 \pm 100 M_{\odot} \mathrm{yr}^{-1}$. Note that these SFRs must be corrected down by the factor $\mu_{\text {lens }}$ to account for lensing magnification. With our preferred best-fit correction factor of $2.2 \pm 0.3$ for the model involving two components to describe $1.1 \mathrm{~mm}$ emission from HFLS3, the 
instantaneous and $100 \mathrm{Myr}$ averaged SFRs are $\sim 1300 M_{\odot} \mathrm{yr}^{-1}$ and $\sim 660 M_{\odot} \mathrm{yr}^{-1}$, respectively. The two are different as the Kennicutt (1998) relation assumes a bolometric luminosity of a constant star formation lasting over $100 \mathrm{Myr}$ emitted in the infrared (Kennicutt 1998; Leitherer \& Heckman 1995). For a constant star formation, bolometric luminosity after the first $10 \mathrm{Myr}$ evolves relatively slowly as the rate of birth and death of massive stars that dominate the bolometric luminosity reach a steady state. For starbursting galaxies, however, the SFR is likely changing rapidly over the $100 \mathrm{Myr}$ time interval, and we may be observing the galaxy at the peak of the SFR. Such a possibility then naturally explains why the instantaneous SFR is a factor of two higher than the SFR averaged over the last 100 Myr.

We can also place a strict lower limit on the SFRs using the $95 \%$ confidence level upper limit on lensing magnification. This leads to values of $>780 M_{\odot} \mathrm{yr}^{-1}$ and $390 M_{\odot} \mathrm{yr}^{-1}$ for instantaneous and $100 \mathrm{Myr}$ averaged SFRs, respectively. This revision of the SFR to a lower value is consistent with a similar revision to the SFR of $z=5.3$ SMG AzTEC-3 (Capak et al. 2011). While the total IR luminosity implies an SFR of $1800 M_{\odot} \mathrm{yr}^{-1}$ (Capak et al. 2011; Riechers et al. 2010), SED modeling of the fluxes with population synthesis models have shown the SFR, averaged over the last $100 \mathrm{Myr}$, to be as low as $500 M_{\odot} \mathrm{yr}^{-1}$ (Dwek et al. 2011). Our SED models also show that the age of the oldest stars in HFLS3 is around $200 \mathrm{Myr}$, suggesting that HFLS3 started assembling its stars at a redshift of $\sim 8$, during the epoch of reionization.

Using the far-IR/submillimeter SED and the standard assumptions used in MAGPHYS and correcting for magnification, the dust mass of HFLS3 is $\sim 3 \times 10^{8} M_{\odot}$, with a lower limit at $2 \times 10^{8} M_{\odot}$. The interstellar medium includes two components with dust temperatures of $24 \pm 2$ and $50 \pm 2 \mathrm{~K}$. The best-fit SED model is such that $>90 \%$ of the dust mass is in the warm phase, contrary to low-redshift star-forming galaxies that have a lower ratio. Such a high ratio for HFLS3 establishes that most of the dust is associated with starbursting clumps and not the diffuse cirrus. The implied dust temperature of the cold phase component is comparable to the cosmic microwave background (CMB) temperature at $z=6.3$, suggesting that the extended cirrus of this galaxy may be in radiative equilibrium with the CMB. Using the Chabrier (2003) IMF, with parameters derived again from the SED fits using MAGPHYS and with lensing magnification included, we find that HFLS3 has a stellar mass of about $5 \times 10^{10} M_{\odot}$. This stellar mass, however, is highly uncertain as it is based on just three detections at the rest-frame UV wavelengths. In all of these cases, the detections are at the level of $3 \sigma$. Furthermore, we have assumed that the magnification factor derived with $1.1 \mathrm{~mm}$ continuum map also applies for the rest-frame UV emission from which the stellar mass is derived. Regardless of these uncertainties, we find that HFLS3 has formed a substantial amount of stellar mass already. Such a high stellar mass is already at the limits allowed by the dynamical mass of HFLS3 reported in Riechers et al. (2013).

While the SED-based stellar mass is uncertain by an order of magnitude once all modeling errors are accounted for, the dust mass of HFLS3 with a value $\sim 3 \times 10^{8} M_{\odot}$ provides an additional constraint on the stellar mass of HFLS3. This comes from models related to the dust formation mechanisms in massive starbursts where core-collapse supernovae (CCSNe) are expected to be the origin of the bulk of the elements that formed the dust. The contribution of low-mass stars to the refractory elements is negligible in a young galaxy such as HFLS3. Thus, the total number of CCSNe that exploded in the galaxy dictates the maximum dust mass. Following the arguments in D. Watson et al. (2014, in preparation), from an observed dust mass, we can infer the minimum number of supernovae that occurred and for a particular IMF, the resulting lower bound on the stellar mass. The simplest and most robust way to make such an estimate on the stellar mass is to work from observations. SN 1987A is close to the mass-integrated mean CCSN mass for most IMFs and is the best-observed CCSN remnant known. Assuming SN 1987A as a good mass-weighted mean for the dust production, and using the preferred value of a carbonaceous and silicate grain mix of 0.6-0.7 $M_{\odot}$ (Matsuura et al. 2011; Indebetouw et al. 2014), we can infer that at least $2 \times 10^{8} M_{\odot}$ CCSNe exploded in HFLS3 to account for the dust mass of $3 \times 10^{8} \mathrm{M}_{\odot}$.

The stellar-to-dust mass ratio should be around 100 for a Chabrier IMF and a factor of two larger than this for a Salpeter IMF. The precise value of this ratio depends on how CCSNe produce refractory metals as a function of mass. When considering model uncertainties, the stellar-to-dust mass ratio is within $20 \%$ of the value quoted above, where the dust masses are tied through the observational pivot point provided by the dust mass observed in SN 1987A. Note that this argument is currently based on the dust mass observed in SN 1987A as an indication of the refractory element production, rather than claiming that CCSNe necessarily produce all the dust directly. However, since the dust mass observed is believed to be close to the maximal dust production for this SN (Indebetouw et al. 2014), it is therefore a reasonable reflection of the most dust we could ultimately expect to be produced by the elements synthesized by SN 1987A. Thus, for $3 \times 10^{8} M_{\odot}$ mass of dust in the galaxy, we expect a minimum of $\sim 2 \times 10^{10} M_{\odot}$ mass of stars for a Chabrier IMF and twice this for a Salpeter IMF. This is comparable to the lensing magnification-corrected stellar mass inferred from MAGPHYs at $5 \times 10^{10} M_{\odot}$, though we note once again that this value has a large uncertainty due to various assumptions and a low signal-to-noise ratio of the restframe UV measurements. For an SFR averaged over $100 \mathrm{Myr}$ of about $660 M_{\odot} \mathrm{yr}^{-1}$, the above arguments imply a characteristic dust production time of at least $40 \mathrm{Myr}$, assuming a negligible dust destruction during the same period. This is lower than the suggested lifetime for dust mass assembly in AzTEC-3 of about 200 Myr (Dwek et al. 2011). While our current estimates are uncertain, the above argument, however, can be strengthened in the future with more precise measurements of dust and stellar masses to constrain dust production mechanisms at $z \sim 6$.

We also attempted a SED model with far-IR/submillimeter data points combined with rest-UV fluxes from R1, with peak emission 0.'5 to the southwest of HFLS3 (Figure 1). This emission is detected in all three WFC3 bands at significances greater than $6 \sigma$ in each, although the emission remains undetected in the ACS. The emission, however, is blended in IRAC data with the near-IR emission from the two galaxies (Figure 4). The MAGPHYs fit was considerably poor as there was no consistent SED that can fit the four orders of magnitude in wavelength from UV to submillimeter in that case with the best-fit case having a reduced $\chi^{2}$ of greater than five. This ruled out a scenario in which HFLS3 submillimeter emission is associated with R1. It also rules out an extreme scenario in which our relative astrometry between IRAM/PdBI and Hubble images are wrong such that the near-IR counterpart to HFLS3 is R1. We find that R1 must be a separate source. The HYPERz SED model shown in Figure 5 leads to a photometric redshift for this emission that is consistent with a source at $z \sim 6.3$ (Figure 2, bottom panel), 
though a dusty galaxy SED at $z \sim 2$ is also consistent with this emission. The HyPERz fit to the data leads to a stellar mass of $\sim 1.2 \times 10^{10} M_{\odot}$ for R1 if we assume the redshift is $z=6.3$, following the $z \sim 6$ photo- $z$ solution.

We have two possibilities for this new source. It could be part of the emission associated with a complex galaxy merger system involving HFLS3, especially if HFLS3 starburst is triggered by a merger, as is the case for most $z \sim 2$ bright SMGs. Alternatively, it could be part of the $z \sim 2.1$ foreground structure that is responsible for the lensing of HFLS3. If the latter is indeed the case, the region in the foreground of HFLS3 involves a massive galaxy group, but the magnification upper limit of 3.7 we have derived here is unlikely to be revised higher as it accounts for a wide variation of model parameters, including to the total lens mass in the foreground. It is far more likely that R1 is part of the complex merger system associated with HFLS3.

\section{SUMMARY}

Here, we have discussed the rest-frame ultraviolet emission from the starbursting galaxy HFLS3 at a redshift of 6.34. The recently acquired Hubble/WFC3 and ACS imaging data show conclusively that the previously identified rest-frame optical counterpart of HFLS3 is at $z<6$. We find two galaxies in the foreground leading to a clear possibility for lensing magnification, though at a level below that needed to form multiple images. A lensing model based on the Hubble imaging data then leads to a magnification factor for the millimeterwave continuum emission of $2.2 \pm 0.3$, with a strict upper limit of 3.7 at the $95 \%$ confidence level. The scenario involving no lensing is ruled out at more than the $6 \sigma$ confidence level. Using models for the rest-frame UV to far-IR SED, we determine the instantaneous SFR, 100 Myr averaged SFR, dust, and stellar masses of HFLS3 to be $1320 M_{\odot} \mathrm{yr}^{-1}, 660 M_{\odot} \mathrm{yr}^{-1}, 3 \times$ $10^{8} M_{\odot}$, and $5 \times 10^{10} M_{\odot}$, respectively, with large uncertainties especially on the stellar mass of HFLS3. The properties of HFLS3 suggest a galaxy that has intrinsic properties that are roughly consistent with $z=5.3$ SMG AzTEC-3, but there are also differences resulting from the higher dust and stellar mass of HFLS3.

Galaxies with submillimeter colors similar to HFLS3 have been now identified in SPIRE data, leading to the possibility that detailed statistical studies on massive, dusty, starbursts during reionization will become feasible with future facilities (Dowell et al. 2014). While statistical studies will be necessary to address fundamental questions regarding how such massive, metal-rich, starbursting galaxies could form 800 Myr after the Big Bang, detailed studies of individual galaxies are also useful to address whether the astrophysics that govern massive starbursts during reionization are similar to those in $z \sim 2$ SMGs.

Financial support for this work was provided by NASA through grant HST-GO-13045 from the Space Telescope Science Institute, which is operated by Associated Universities for Research in Astronomy, Inc., under NASA contract NAS 5-26555. Additional support for A.C., W.O., J.C., J.L.W., and C.M.C. was from NSF with AST-1313319. We thank E. da Cunha for help with MagPhys. Dark Cosmology Centre is funded by the Danish National Research Foundation (J.L.W. and D.W.). S.O. acknowledges support from the Science and
Technology Facilities Council [grant No. ST/I000976/1]. SPIRE has been developed by a consortium of institutes led by Cardiff Univ. (UK) and including Univ. Lethbridge (Canada); NAOC (China); CEA, LAM (France); IFSI, Univ. Padua (Italy); IAC (Spain); Stockholm Observatory (Sweden); Imperial College London, RAL, UCL-MSSL, UKATC, Univ. Sussex (UK); and Caltech, JPL, NHSC, Univ. Colorado (USA). This development has been supported by national funding agencies: CSA (Canada); NAOC (China); CEA, CNES, CNRS (France); ASI (Italy); MCINN (Spain); SNSB (Sweden); STFC, UKSA (UK); and NASA (USA). The data presented in this paper will be released through the Herschel Database in Marseille HeDaM (hedam.oamp.fr/HerMES).

\section{REFERENCES}

Blain, A. W. 1996, MNRAS, 283, 1340

Bolzonella, M., Miralles, J.-M., \& Pelló, R. 2000, A\&A, 363, 476 Bruzual, G., \& Charlot, S. 2003, MNRAS, 344, 1000

Calzetti, D., Armus, L., Bohlin, R. C., et al. 2000, ApJ, 533, 682

Capak, P. L., Riechers, D., Scoville, N. Z., et al. 2011, Natur, 470, 233

Casey, C. M., Narayanan, D., \& Cooray, A. 2014, PhR, in press

Chabrier, G. 2003, ApJL, 586, L133

Chapman, S. C., Blain, A. W., Smail, I., \& Ivison, R. J. 2005, ApJ, 622, 772

Combes, F., Rex, M., Rawle, T. D., et al. 2012, A\&A, 538, L4

Coppin, K. E. K., Chapman, S. C., Smail, I., et al. 2010, MNRAS, 407, L103

da Cunha, E., Charlot, S., \& Elbaz, D. 2008, MNRAS, 388, 1595

Daddi, E., Dannerbauer, H., Stern, D., et al. 2009, ApJ, 694, 1517

Dowell, C. D., Conley, A., Glenn, J., et al. 2014, ApJ, 780, 75

Dwek, E., Staguhn, J. G., Arendt, R. G., et al. 2011, ApJ, 738, 36

Fruchter, A. S., Hack, W., Dencheva, N., et al. 2010, in 2010 Space Telescope Science Institute Calibration Workshop, ed. S. Deustua \& C. Oliviera (Baltimore, MD: STScI), 382

Geach, J. E., Smail, I., Chapman, S. C., et al. 2007, ApJL, 655, L9

Griffin, M. J., Abergel, A., Abreu, A., et al. 2010, A\&A, 518, L3

Hainline, L. J., Blain, A. W., Smail, I., et al. 2011, ApJ, 740, 96

Hezaveh, Y. D., Marrone, D. P., \& Holder, G. P. 2012, ApJ, 761, 20

Hinshaw, G., Larson, D., Komatsu, E., et al. 2013, ApJS, 208, 19

Indebetouw, R., et al. 2014, ApJL, 782, L2

Keeton, C. R. 2001, arXiv:astroph/012340

Kennicutt, R. C., Jr. 1998, ApJ, 498, 541

Leitherer, C., \& Heckman, T. M. 1995, ApJS, 96, 9

Lo Faro, B., Franceschini, A., Vaccari, M., et al. 2013, ApJ, 762, 108

Madau, P. 1995, ApJ, 441, 18

Matsuura, M., Dwek, E., Meixner, M., et al. 2011, Sci, 333, 1258

Negrello, M., Hopwood, R., De Zotti, G., et al. 2010, Sci, 330, 800

Negrello, M., Perrotta, F., González-Nuevo, J., et al. 2007, MNRAS, 377, 1557

Oliver, S. J., Bock, J., Altieri, B., et al. 2012, MNRAS, 424, 1614

Paciga, G., Scott, D., \& Chapin, E. L. 2009, MNRAS, 395, 1153

Peng, C. Y., Ho, L. C., Impey, C. D., \& Rix, H.-W. 2002, AJ, 124, 266

Perrotta, F., Baccigalupi, C., Bartelmann, M., De Zotti, G., \& Granato, G. L. 2002, MNRAS, 329, 445

Pilbratt, G. L., Riedinger, J. R., Passvogel, T., et al. 2010, A\&A, 518, L1 Planck Collaboration, et al. 2013, arXiv:1303.5076

Riechers, D. A., Bradford, C. M., Clements, D. L., et al. 2013, Natur, 496, 329

Riechers, D. A., Capak, P. L., Carilli, C. L., et al. 2010, ApJL, 720, L131

Robson, E. I., Ivison, R. J., Smail, I., et al. 2014, arXiv:1403.0247

Serjeant, S. 2012, MNRAS, 424, 2429

Smail, I., Chapman, S. C., Blain, A. W., \& Ivison, R. J. 2004, ApJ, 616, 71

Swinbank, A. M., Smail, I., Chapman, S. C., et al. 2010, MNRAS, 405, 234

Vieira, J. D., Marrone, D. P., Chapman, S. C., et al. 2013, Natur, 495, 344

Walter, F., Decarli, R., Carilli, C., et al. 2012, Natur, 486, 233

Wardlow, J. L., Cooray, A., De Bernardis, F., et al. 2013, ApJ, 762, 59

Wardlow, J. L., Smail, I., Coppin, K. E. K., et al. 2011, MNRAS, 415, 1479

Weiß, A., De Breuck, C., Vieira, J. D., et al. 2013, in ASP Conf. Ser. 476, New Trends in Radio Astronomy in the ALMA Era: The 30th Anniversary of Nobeyama Radio Observatory, ed. R. Kawabe, N. Kuno, \& S. Yamamoto (San Francisco, CA: ASP), 33

Wyithe, J. S. B., Yan, H., Windhorst, R. A., \& Mao, S. 2011, Natur, 469, 181 